\title{
GABAergic Excitation in the Basolateral Amygdala
}

\author{
Alan R. Woodruff, ${ }^{1}$ Hannah Monyer, ${ }^{2}$ and Pankaj Sah ${ }^{1}$ \\ ${ }^{1}$ Queensland Brain Institute and School of Biomedical Sciences, University of Queensland, 4072 Brisbane, Australia, and ${ }^{2}$ Interdisciplinary Centre for \\ Neurosciences, University of Heidelberg, 69120 Heidelberg, Germany
}

\begin{abstract}
GABA-containing interneurons are a diverse population of cells whose primary mode of action in the mature nervous system is inhibition of postsynaptic target neurons. Using paired recordings from parvalbumin-positive interneurons in the basolateral amygdala, we show that, in a subpopulation of interneurons, single action potentials in one interneuron evoke in the postsynaptic interneuron a monosynaptic inhibitory synaptic current, followed by a disynaptic excitatory glutamatergic synaptic current. Interneuron-evoked glutamatergic events were blocked by antagonists of either AMPA/kainate or $\mathrm{GABA}_{\mathrm{A}}$ receptors, and could be seen concurrently in both presynaptic and postsynaptic interneurons. These results show that single action potentials in a GABAergic interneuron can drive glutamatergic principal neurons to threshold, resulting in both feedforward and feedback excitation. In interneuron pairs that both receive glutamatergic inputs after an interneuron spike, electrical coupling and bidirectional GABAergic connections occur with a higher probability relative to other interneuron pairs. We propose that this form of GABAergic excitation provides a means for the reliable and specific recruitment of homogeneous interneuron networks in the basal amygdala.
\end{abstract}

Key words: axoaxonic; fear; network; learning; excitation; depolarization

\section{Introduction}

GABAergic neurons are local circuit inhibitory cells that exhibit diverse morphological, biochemical, molecular, and synaptic properties (Freund and Buzaki, 1996; McBain and Fisahn, 2001; Markram et al., 2004; Somogyi and Klausberger, 2005). For these cells, the anatomical location of the GABAergic synapses on the postsynaptic cell in large part determines the functional impact of interneuronal activity on the target neuron. Thus, interneurons targeting perisomatic regions are strategically placed to modulate somatic action potential generation (Cobb et al., 1995; Miles et al., 1996), whereas those terminating more distally may prevent dendritic action potentials (Miles et al., 1996), modulate the induction of glutamatergic synaptic plasticity (Magee and Johnston, 1997), or gate the transfer of information along the somatodendritic axis (Buzsaki et al., 1996). At the network level, these effects are facilitated by the presence of chemically and electrically connected networks of homogeneous interneuron populations (Galarreta and Hestrin, 1999; Gibson et al., 1999; Hestrin and Galarreta, 2005), allowing widespread control of glutamatergic principal neuron activity.

Despite this diversity, all inhibitory interneurons above the brainstem release GABA at their synaptic terminals, activating ionotropic, chloride-permeable $\mathrm{GABA}_{\mathrm{A}}$ receptors. In immature neurons, the transmembrane chloride gradient favors chloride efflux and results in cell depolarization (Ben-Ari, 2002). With development, increased expression of the $\mathrm{K}^{+}-\mathrm{Cl}^{-}$cotransporter KCC2 leads to a decrease in intracellular chloride concentration,

Received Aug. 6, 2006; revised 0ct. 8, 2006; accepted 0ct. 9, 2006.

This work was supported by grants from the National Health and Medical Research Council of Australia and the Australian Research Council.

Correspondence should be addressed to Pankaj Sah, Queensland Brain Institute, University of Queensland, Brisbane, Queensland 4072, Australia. E-mail: pankaj.sah@uq.edu.au.

D01:10.1523/JNEUROSCI.3389-06.2006

Copyright $\odot 2006$ Society for Neuroscience $\quad$ 0270-6474/06/2611881-07\$15.00/0 creating a more hyperpolarized chloride reversal potential $\left(E_{\mathrm{Cl}}\right)$, thus switching GABAergic effects from depolarizing to hyperpolarizing (Rivera et al., 1999). As a result, interneurons in the mature brain are thought to play a primarily inhibitory role in synaptic integration by shunting excitation. However, exceptions to a hyperpolarizing role for GABA have been described under both pathological (Cossart et al., 2005) and physiological (Marty and Llano, 2005) conditions. Most instances of depolarizing GABA actions during nonpathological states have been reported after intense or prolonged activation of $\mathrm{GABA}_{\mathrm{A}}$ receptors (Alger and Nicoll, 1979, 1982; Staley et al., 1995; Kaila et al., 1997), and are thought to result from a breakdown of the chloride gradient, followed by efflux of bicarbonate anions through open $\mathrm{GABA}_{\mathrm{A}}$ channels (Staley et al., 1995). However, two recent studies have reported increased postsynaptic spiking after single-pulse stimulation of GABAergic neurons in adult cerebellum (Chavas and Marty, 2003) and cortex (Gulledge and Stuart, 2003).

Here, we describe a potent excitatory effect of a subpopulation of GABAergic interneurons in the basal nucleus of the amygdala (BL). Using mice that express enhanced green fluorescent protein (EGFP) under the control of the parvalbumin promoter (Meyer et al., 2002), we show that a single action potential in a parvalbumin expressing $\left(\mathrm{PV}^{+}\right)$interneuron can evoke a rapid, biphasic, outward/inward current sequence in postsynaptic $\mathrm{PV}^{+}$interneurons. Outward currents are monosynaptic GABAergic events, whereas inward currents are disynaptic and caused by activation of AMPA receptors, suggesting the recruitment of glutamatergic neurons. Using paired recordings from interneuron pairs, we further show that interneurons connected by electrical or GABAergic synapses receive simultaneous excitation from activated principal neurons. We therefore propose that this form of GABAergic excitation will allow the synchronous recruitment of interneuron networks. 


\section{Materials and Methods}

Electrophysiology. All procedures were conducted in accordance with the Australian Code of Practice for the Care and Use of Animals for Scientific Purposes, and were approved by the University of Queensland Animal Ethics Committee. Coronal brain slices containing basolateral amygdala were made from 16- to 25-d-old mice that expressed EGFP under the control of the parvalbumin promoter (Meyer et al., 2002). Heterozygous PV-EGFP animals were bred with BALB/c mice and PV-EGFP ${ }^{+}$animals identified by examining the fluorescence in skeletal muscle on their ears using a UV lamp. After halothane anesthesia, animals were decapitated, and the brain was rapidly removed and placed in ice-cold, oxygenated artificial CSF (aCSF) containing the following (in $\mathrm{mm}$ ): $118 \mathrm{NaCl}, 2.5$ $\mathrm{KCl}, 25 \mathrm{NaHCO}_{3}, 10$ glucose, $1.3 \mathrm{MgCl}_{2}, 2.5 \mathrm{CaCl}_{2}$, and $1.2 \mathrm{NaH}_{2} \mathrm{PO}_{4}$. Slices $(300 \mu \mathrm{m})$ were cut on a vibratome (Dosaka, Kyoto, Japan) and incubated at $32^{\circ} \mathrm{C}$ for $30 \mathrm{~min}$ before being allowed to equilibrate at room temperature for at least an additional $30 \mathrm{~min}$.

During recording, slices were perfused with heated aCSF $\left(34 \pm 2^{\circ} \mathrm{C}\right)$. Recording pipettes (3-5 $\mathrm{M} \Omega$ ) fabricated from borosilicate glass were filled with an intracellular solution containing the following (in mM): 135 $\mathrm{KMeSO}_{4}, 8 \mathrm{NaCl}, 10$ HEPES, $2 \mathrm{Mg}_{2} \mathrm{ATP}, 0.3 \mathrm{Na}_{3} \mathrm{GTP}$, 0.1 spermine, 7 phosphocreatine, and 0.3 EGTA. In some experiments, neurobiotin $(0.2 \%)$ was also included in the internal solution. PV-EGFP neurons were visualized using an upright microscope (Olympus BX50WI; Olympus Optical, Tokyo, Japan) equipped with a fluorescence attachment. Paired whole-cell recordings were made using Axopatch 1D and Axopatch 200B amplifiers (Molecular Devices, Foster City, CA). Signals were filtered at $5 \mathrm{kHz}$ and digitized at $10 \mathrm{kHz}$ using an ITC-16 board (InstruTech, Port Washington, NY). Data acquisition and analysis were performed using Axograph (Axograph Scientific).

Cells included in this study were restricted to those exhibiting an initial resting membrane potential more hyperpolarized than $-55 \mathrm{mV}$. Immediately after breakthrough, cells were injected with $600 \mathrm{~ms}$ current pulses ( -100 to $700 \mathrm{pA} ; 50 \mathrm{pA}$ increments) to confirm interneuron identity. Presynaptic interneurons were injected with a $2 \mathrm{~ms}, 2 \mathrm{nA}$ current to evoke an action potential. For voltage-clamp recordings, the postsynaptic cell was held at $-60 \mathrm{mV}$ for AMPA/kainate currents and at $-40 \mathrm{mV}$ for $\mathrm{GABA}_{\mathrm{A}}$ currents, unless otherwise indicated. IPSC and EPSC latency was measured from the peak of the presynaptic action potential to the onset of the postsynaptic event. Spontaneous synaptic events were detected using the template detection software in Axograph. Amplitudes of IPSCs and EPSCs were calculated by averaging all responses (excluding failures).

Immunocytochemistry. For neurons filled with neurobiotin, slices were fixed overnight in $4 \%$ paraformaldehyde and resectioned at $100 \mu \mathrm{m}$. Neurons were visualized using the avidin-biotinylated horseradish peroxidase reaction with nickel intensification (Vectastain ABC Elite kit; Vector Laboratories, Burlingame, CA). Slices were soaked overnight in avidin-horseradish peroxidase. After washing in Tris buffer $(0.1 \mathrm{M} ; \mathrm{pH}$ 7.4), filled cells were visualized using the diaminobenzidine procedure. Slices were then mounted on albumin-coated slides, dried overnight, dehydrated in an ascending series of alcohols, cleared in xylene and coverslipped in Permount. For immunohistochemistry, slices were permeabilized in blocking buffer (PBS plus $0.1 \%$ Triton X-100, 2\% fetal calf serum, $2 \%$ goat serum, $2 \% \mathrm{BSA}$ ) for $30 \mathrm{~min}$, and then incubated overnight at $4{ }^{\circ} \mathrm{C}$ with a mouse monoclonal parvalbumin antibody (Sigma-Aldrich, St. Louis, MO) (1:2000 dilution in blocking buffer). Incubated slices were washed three times for $15 \mathrm{~min}$ in PBS, and then incubated at room temperature for $1 \mathrm{~h}$ in Alexa Fluor 350-conjugated goat anti-mouse $\operatorname{IgG}(\mathrm{H}+\mathrm{L} ; 1: 1000$ in blocking buffer; Invitrogen, San Diego, CA) and streptavidin Alexa Fluor-568 (1:500 in blocking buffer; Invitrogen). Slices were washed three times for $15 \mathrm{~min}$ in PBS, and then briefly in distilled water before being transferred to slides, where they were mounted in fluorescent mounting medium (DakoCytomation, Carpinteria, CA), coverslipped, and analyzed using an upright fluorescence microscope (Zeiss Axioplan 2; Zeiss, Göttingen, Germany).

\section{Results}

\section{Interneurons evoke feedforward glutamatergic excitation}

Simultaneous dual recordings were made from 162 pairs of $\mathrm{PV}^{+}$ interneurons (Fig. 1a). In the majority of synaptically connected pairs $(n=46)$, single action potentials in the presynaptic neuron evoked a synaptic current in the voltage-clamped postsynaptic neuron that occurred with fixed latency, reversed near the chloride equilibrium potential (Fig. $1 b$ ), and was blocked by the $\mathrm{GABA}_{\mathrm{A}}$ receptor antagonist bicuculline $(10 \mu \mathrm{M} ; n=4)$, confirming it as an IPSC. However, in 10 pairs, a single presynaptic action potential evoked a delayed inward current in the postsynaptic interneuron. In 6 of these 10 pairs, an outward current preceded the inward current, resulting in a biphasic outward-inward current sequence at a holding potential of $-40 \mathrm{mV}$ (Fig. 1c). As for the GABAergic IPSC, the outward current occurred with a fixed latency. In contrast, the inward current occurred with a fluctuating delay and was unreliable relative to the IPSC, displaying many failures. In cases in which both outward and inward components were present, shunting of the IPSC was clearly evident in the average trace (Fig. 1c). Hyperpolarization of the postsynaptic cell to $-90 \mathrm{mV}$ reversed the initial outward current, as expected for a GABAergic IPSC, and increased the amplitude of the delayed inward current (Fig. 1d).

At a holding potential of $-40 \mathrm{mV}$, the average latency of the IPSC was $0.50 \pm 0.06 \mathrm{~ms}(n=6)$ and had a SD (synaptic jitter) of $0.23 \pm 0.07 \mathrm{~ms}(n=6)$ (Fig. $2 a)$. In comparison, the inward current had a significantly larger average latency $(2.9 \pm 0.3 \mathrm{~ms}$; $n=8 ; p<0.001)$ and $\mathrm{SD}(0.51 \pm 0.07 \mathrm{~ms} ; n=8, p<0.02)$ (Fig. $2 b)$. Application of the AMPA/kainate receptor antagonist 6-nitro-7-sulfamoylbenzo(f)quinoxaline-2,3-dione (NBQX) (10 $\mu \mathrm{M})$ selectively abolished the delayed inward current $(n=5)$ and the remaining outward current could subsequently be blocked by the $\mathrm{GABA}_{\mathrm{A}}$ receptor antagonist bicuculline (Fig. $2 c$ ). The NBQXsensitive component, obtained by digital subtraction, had a decay time constant of $2.8 \pm 0.5 \mathrm{~ms}(n=5)$, similar to that of spontaneous EPSCs in interneurons $(2.3 \pm 0.2 \mathrm{~ms} ; n=14 ; p>0.05)$ (Mahanty and Sah, 1998).

These data show that single action potentials in $\mathrm{PV}^{+}$interneurons can evoke a monosynaptic, time-locked GABAergic IPSC followed by a delayed, fluctuating glutamatergic EPSC in another $\mathrm{PV}^{+}$interneuron.

What is the origin of this EPSC? As parvalbumin-positive interneurons make extensive inhibitory connections onto local principal neurons (Smith et al., 1998), GABAergic inhibition of an intermediary principal neuron may be followed by a rebound action potential in the glutamatergic cell (Cobb et al., 1995), causing release of glutamate onto the postsynaptic interneuron. However, the relatively rapid latency of the EPSC $(2.5 \mathrm{~ms})$ is inconsistent with this possibility because IPSPs recorded in principal neurons lasted $>50 \mathrm{~ms}$ (A. R. Woodruff and P. Sah, unpublished observations). A second possibility is that, as for mossy fibers in the hippocampus (Ruiz et al., 2003), GABA and glutamate may be coreleased at some synapses of parvalbuminexpressing neurons, with glutamate release being delayed and asynchronous. However, this is unlikely because application of bicuculline blocked both the GABAergic IPSC and the inward AMPA current $(n=4)$ (Figs. $2 c, 5 a)$. We therefore suggest that the delayed fluctuating EPSC arises through direct GABAergic excitation of a local glutamatergic neuron to threshold that in turn innervates the postsynaptic interneuron. The delayed and variable onset of the inward current is consistent with its disynaptic nature (Pouille and Scanziani, 2001). 
$\mathbf{a}$
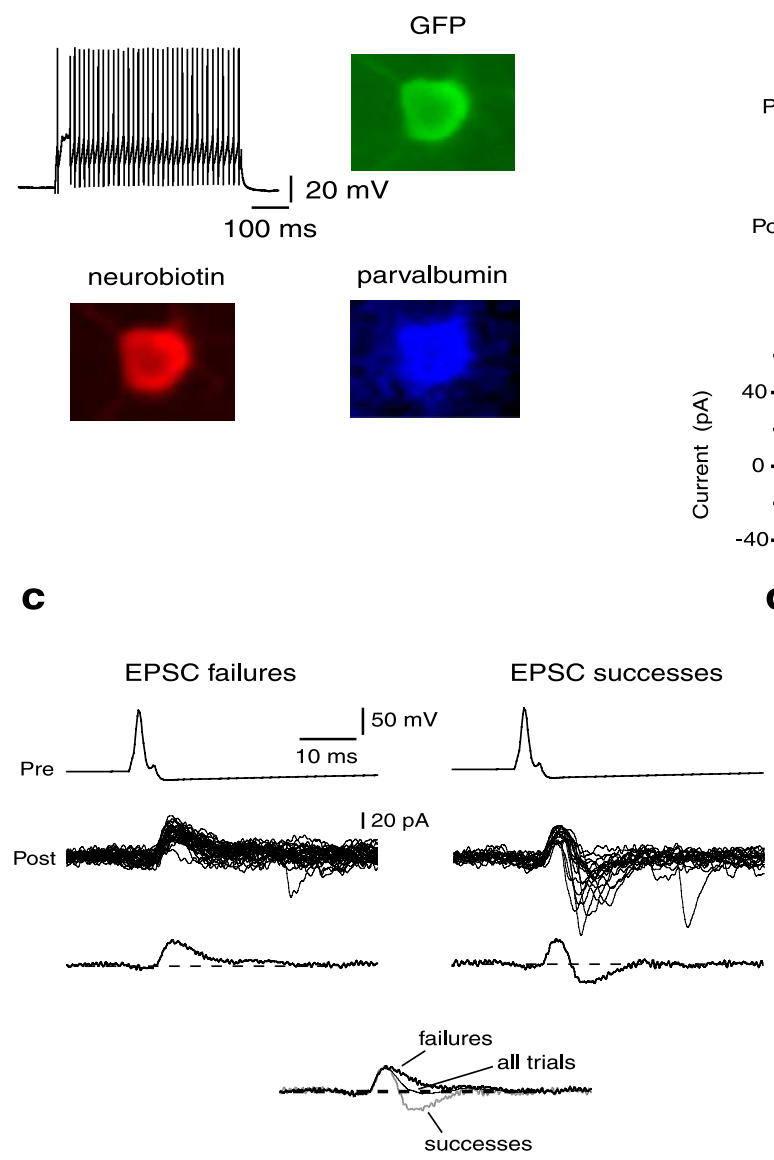

Figure 1. Single interneuron action potentials evoke biphasic currents in postsynaptic interneurons. $\boldsymbol{a}$, GFP-expressing neurons show electrophysiological responses typical of interneurons. Illustrated is the voltage response to a $600 \mathrm{~ms}$ suprathreshold, depolarizing current injection. All recorded neurons expressed GFP (green). In some recordings $(n=4)$, neurons were loaded with neurobiotin (red). These neurons were positive for parvalbumin (blue), confirming they are parvalbumin-expressing interneurons. $\boldsymbol{b}$, Paired recordings from two EGFP-positive interneurons (top). Traces below show the current response in the voltage-clamped postsynaptic cell at holding potentials of -40 and $-90 \mathrm{mV}$ (bottom traces) to a single action potential in the presynaptic interneuron (top trace). The current-voltage relationship of the postsynaptic current is shown in the bottom panel. c, Interneuron pairs show biphasic currents. Single action potentials evoked in the presynaptic interneuron evoke two types of responses in a voltage-clamped postsynaptic interneuron $\left(V_{h},-40 \mathrm{mV}\right)$. Traces on the left show time-locked outward current responses, whereas traces on the right show responses that are also accompanied by a delayed inward current. The average current in each case is shown in the bottom trace. The bottom panel shows overlay of averaged traces in which there were EPSC failures (thick black), EPSC successes (gray), or the average for all trials (thin black). Note the faster kinetics of the synaptic current when all trials are averaged. $\boldsymbol{d}, 0$ nly the early synaptic current reverses at the chloride equilibrium potential. Averaged postsynaptic responses in a voltage-clamped postsynaptic interneuron to a single action potential in the presynaptic interneuron. Note the dual component response. Hyperpolarization of the postsynaptic neuron to $-90 \mathrm{mV}$ reversed the initial component and increased the amplitude of the delayed inward current. The shaded region is expanded at the bottom to demonstrate the delay of the large inward component.

\section{Interneurons evoke feedback glutamatergic excitation}

We have shown that single action potentials in some $\mathrm{PV}^{+}$interneurons can evoke a disynaptic feedforward EPSC in a nearby $\mathrm{PV}^{+}$interneuron. In a population of $\mathrm{PV}^{+}$interneurons, feedback excitation was also apparent, so that single action potentials in these cells were followed by an EPSP (Fig. 3a). These EPSPs increased in amplitude with hyperpolarization, and were blocked by NBQX $(n=3)$, confirming that they were glutamatergic. They were also abolished by bicuculline (see Fig. $5 a$ ), indicating they were feedback EPSPs that arose via direct GABAergic depolarization of a BL glutamatergic neuron. To examine the properties of this feedback excitation, we voltage clamped interneurons at a holding potential of $-70 \mathrm{mV}$. Delivery of a brief depolarizing voltage step $(70 \mathrm{mV} ; 0.5 \mathrm{~ms})$ resulted in an unclamped "action current" that was followed by a feedback EPSC (Fig. 3b). Trials in which EPSCs were not present (failures) were averaged, and individual trials of EPSC successes subtracted from the failure average to reveal the pure feedback EPSC (Fig. 3b). The average amplitude of this feedback EPSC was $75.4 \pm 18.1 \mathrm{pA}(n=$ 7), considerably larger than the amplitude of spontaneous EPSCs recorded in the same neurons (29.5 $\pm 1.3 \mathrm{pA} ; n=7$ ) (Fig. $3 c$ ). As for the feedforward EPSC, this EPSC displayed a high failure rate and fluctuated in latency (Fig. $3 b, c$ ). Because of this large fluctuation in latency, averaged feedback EPSCs displayed a noticeably slower $10-90 \%$ rise time than the averaged spontaneous EPSCs (Fig. 3c). However, both the rise times and decay time constant of individual feedback EPSCs were similar to those of spontaneous EPSCs (Fig. 3d,e) showing that the slow rise time of the averaged feedback EPSC results from the onset jitter of the underlying events and is not attributable to a different receptor mechanism or preferential distal location of feedback EPSCs.

Recently, cortical axoaxonic interneurons (AACs) that make "chandelier" synapses on the axon initial segment of pyramidal neurons (Howard et al., 2005) have been found to depolarize local pyramidal neurons because of a positively shifted $\mathrm{GABA}_{\mathrm{A}}$ reversal potential at the axon initial segment (Szabadics et al., 2006). Chandelier synapses have also been described in the amygdala, and these terminals are known to express parvalbumin (Kemppainen and Pitkanen, 2000; McDonald and Betette, 2001; Muller et al., 2006). Because cortical AAC interneurons express parvalbumin (Howard et al., 2005), we tested whether interneurons that provide disynaptic excitation in the amygdala may also make chandelier synapses. We therefore reconstructed interneurons that showed feedback excitation (Fig. 4a) after loading them with the marker neurobiotin (see Materials and Methods). Reconstructions of interneurons that displayed feedback EPSCs $(n=4)$ revealed that, in all such cells, rows of closely spaced boutons, termed cartridges (Kemppainen and Pitkanen, 2000; McDonald and Betette, 2001), could be observed (Fig. $4 c-f$ ). In addition, all such cells also exhibited rings of boutons that are characteristic of basket cells (McDonald and Betette, 2001) (Fig. 4e,f), indicating that these interneurons make both basket and cartridge synapses. As described previously in the BL (McDonald and Betette, 2001), pericellular baskets could be seen that appear to be continuous with an axonal cartridge (Fig. 4e). We therefore suggest that, as in the cortex, cartridge synapses of parvalbumin-expressing interneurons, which terminate on the axon initial segment of local glutamatergic neurons (Muller et al., 2005), are the likely source of GABAergic excitation in the basal amygdala. 
a

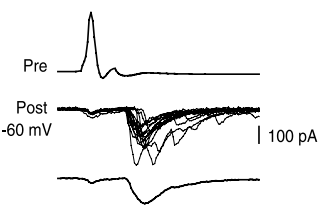

\section{b}

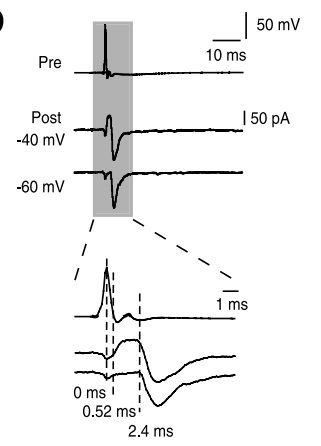

C

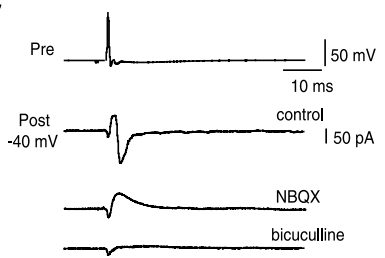

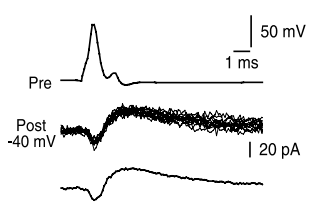
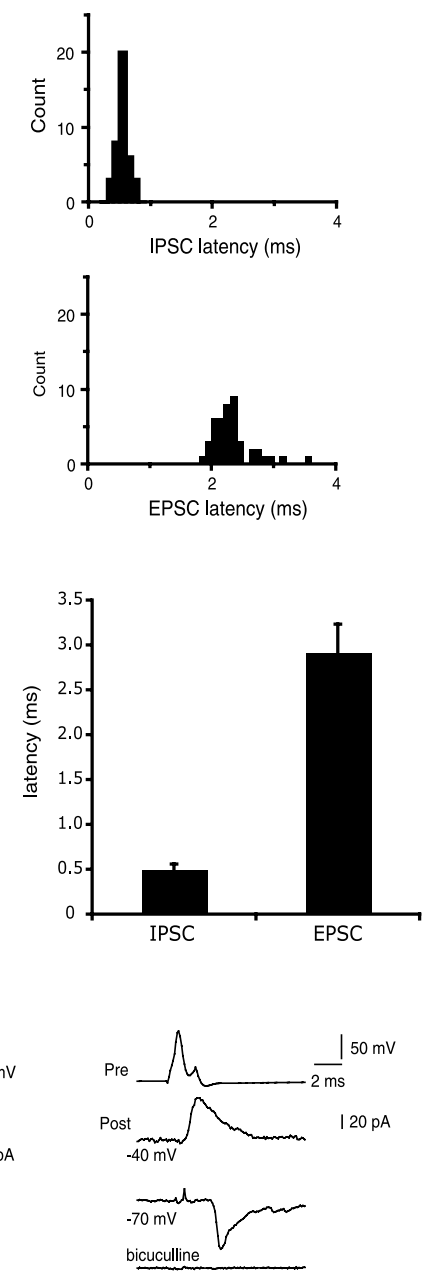

Figure 2. Delayed inward current is disynaptic and glutamatergic. $\boldsymbol{a}$, The latency of the delayed inward current shows a larger variability. Top panels show overlaid fast-latency outward responses at a holding potential of $-40 \mathrm{mV}$ to a single action potential in the presynaptic neuron. The bottom panel shows overlaid delayed inward current responses recorded at a holding potential of $-60 \mathrm{mV}$ to an action potential in the presynaptic neuron. Averaged traces are shown below. The small inward current in the postsynaptic neuron simultaneous with the presynaptic action potentials is attributable to transmission of the action potential via a connected gap junction. The panels on the right show histogram of latencies. $\boldsymbol{b}$, Average responses to single action potentials in the presynaptic neuron recorded at $-40 \mathrm{mV}$ and near the chloride reversal potentials $(-60 \mathrm{mV})$. The shaded region is shown below at an expanded time base. Mean latency of the inward current was significantly longer than for the outward component. Average data for mean latencies is shown on the histogram on the right $(n=8)$. c, Disynaptic current is mediated by GABAergic excitation of a glutamatergic principal neuron. Disynaptic current recorded in a postsynaptic cell voltage clamped at $-40 \mathrm{mV}$ in response to single action potentials in the presynaptic neuron. NBQX $(10 \mu \mathrm{M})$ selectively blocks the inward current, whereas subsequent application of bicuculline $(10 \mu \mathrm{m})$ abolishes the remaining outward current (left panels). Application of bicuculline (10 $\mu \mathrm{M})$ to a naive slice eliminates both components of the postsynaptic response (right panel).

\section{GABAergic excitation leads to recruitment of} interneuron networks

As shown above, action potentials in some $\mathrm{PV}^{+}$interneurons were followed by feedback excitation of variable amplitude. In 5 of 10 paired recordings, these feedback EPSPs occurred simultaneously with feedforward EPSPs recorded in the postsynaptic interneuron. Both presynaptic and postsynaptic EPSPs were abolished by bicuculline (Fig. $5 a)$. In some pairs $(n=2)$, when the postsynaptic neuron was voltage clamped, feedback EPSPs in the presynaptic neuron were time-locked to the AMPA current recorded in the postsynaptic neuron (Fig. $5 b$ ). Furthermore, in a

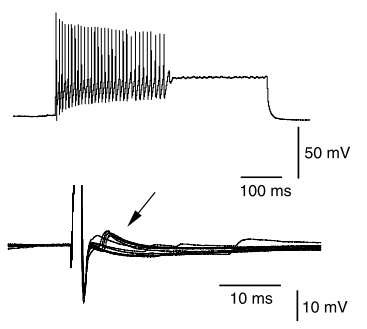

C
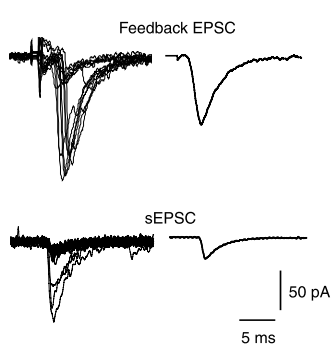

d

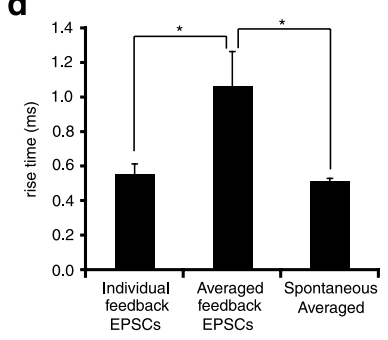

b
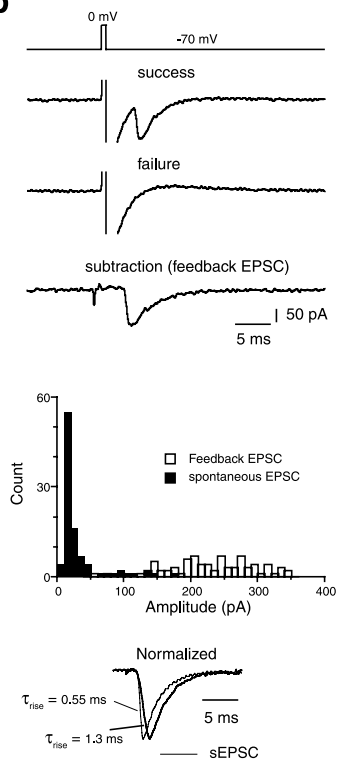

- SEPSC

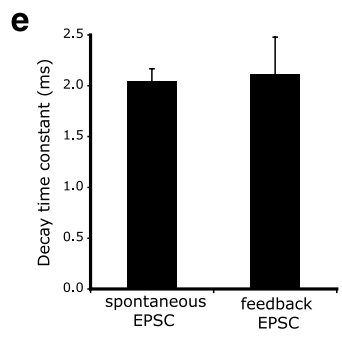

Figure 3. Feedback excitation in parvalbumin-positive interneurons. $\boldsymbol{a}$, Firing phenotype (top left) of an interneuron in response to a $600 \mathrm{~ms}$ depolarizing suprathreshold current injection. The bottom traces show superimposed single action potentials evoked by a brief $(2 \mathrm{~ms})$ current injection, which were followed by feedback EPSPs (arrow). $\boldsymbol{b}$, When the neuron is voltage clamped at $-70 \mathrm{mV}$, a brief $(0.5 \mathrm{~ms})$ voltage step to $0 \mathrm{mV}$ leads to an unclamped "action current" (truncated) that results in GABA release and a feedback EPSC. Trials in which EPSCs were not observed (failures) were averaged, and individual trials of EPSCS subtracted from the failure average to reveal pure feedback EPSCs (trace at bottom of panel). c, EPSCS from feedback excitation are larger than spontaneous EPSCs and fluctuate in amplitude. Traces on the left (top panel) are individual sweeps of feedback EPSCs evoked by brief voltage steps in voltage clamp. Average EPSC is shown on the right. Bottom panels show superimposed sweeps of spontaneous EPSCs in the same neuron aligned at their peak. The average spontaneous EPSC is shown on the right. Histogram plots amplitudes of feedback EPSCs and spontaneous EPSCS. Traces at the bottom show normalized evoked and spontaneous EPSCs superimposed; note the slower rise time of the feedback EPSC. $\boldsymbol{d}$, Mean data showing the 10-90\% rise time of averaged but not individual feedback EPSCS were longer than for spontaneous EPSCs because of variability in the onset of feedback EPSCs. ${ }^{*} p<0.05$. $\boldsymbol{e}$, Mean data of decay time constants of feedback EPSC and spontaneous EPSC. Error bars indicate SEM.

these pairs, failure of the inward current in the postsynaptic cell was accompanied with failure of the EPSP in the presynaptic neuron, indicating that the same excitatory neuron is evoking both the presynaptic depolarization and the postsynaptic current. In four of the five pairs $(80 \%)$ that exhibited both feedforward and feedback excitation, the two interneurons were also connected bidirectionally by GABAergic synapses (Fig. $5 c$ ). Of the remaining pairs $(n=5)$ that exhibited only feedforward excitation, none showed bidirectional GABA synapses. In comparison, of all recorded interneuron pairs $(n=162)$, only six were reciprocally connected with GABAergic synapses. Thus, the probability of bidirectional GABAergic connectivity is greatly enhanced in interneuron pairs exhibiting both disynaptic feedfor- 
a
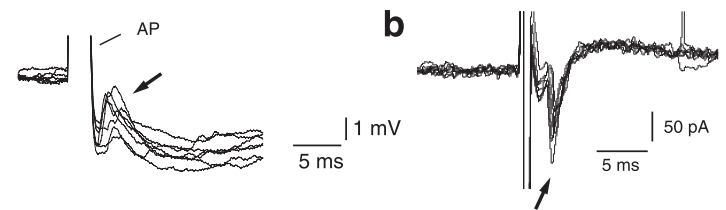

C

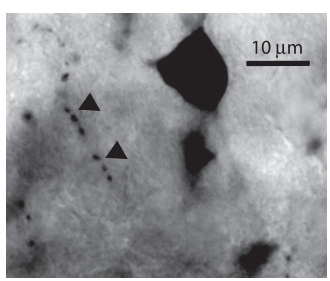

e

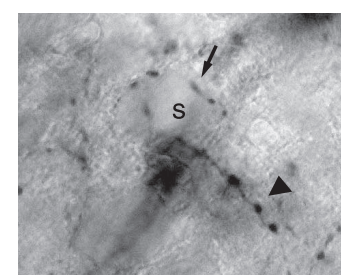

d

f

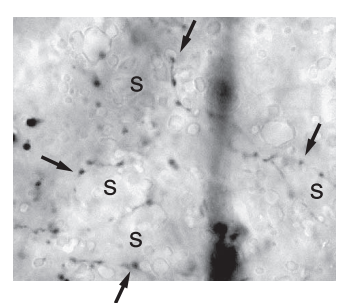

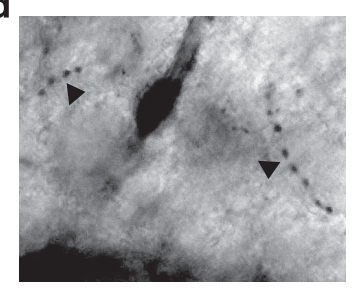

Figure 4. Axoaxonic interneurons produce suprathreshold GABAergic excitation. Shown are recordings from a GFP-positive interneuron that shows feedback excitation. $\boldsymbol{a}$, Single action potentials (APs) shown on an expanded scale have been superimposed. Action potentials are followed by a feedback EPSP (arrow). Action potential peaks have been truncated for clarity. $\boldsymbol{b}$ Under voltage clamp $\left(V_{h},-70 \mathrm{mV}\right)$, a brief $(0.5 \mathrm{~ms})$ voltage step to $0 \mathrm{mV}$ evokes a feedback EPSC (arrow). c, d, Examples of axonal cartridges (arrowheads) in the cell shown in $\boldsymbol{a}$ and $\boldsymbol{b} . \boldsymbol{e}, \boldsymbol{f}$, Cells with feedback excitation possessed axon terminals that formed pericellular baskets (arrows) around the somata (s) of putative principal neurons. Some pericellular baskets (arrow) appear continuous with an axonal cartridge ( $\boldsymbol{e}$, arrowhead).

ward and feedback excitation ( 4 of 5 vs 6 of 162 ; $p \ll 0.001, \chi^{2}$ test). Our data therefore suggest that this GABAergic excitation may be used to recruit interneurons belonging to the same network. Consistent with this, we found that of the five pairs exhibiting both feedforward and feedback excitation, two were also electrically coupled with gap junctions (Fig. $5 d$ ). Once again, this represents a greater than expected probability $(2$ of $5 ; 40 \%)$ than chance (in total, 11 of 162 recorded pairs were electrically coupled; $p<0.01, \chi^{2}$ test).

The recruitment of interneuron networks requires that the glutamatergic activation of interneurons be suprathreshold, either through strong individual synapses or the concerted action of several principal neurons. We confirmed that this could occur both in paired recordings, and in recordings from single interneurons that exhibited feedback excitation (Fig. 5e) in which both feedforward and feedback excitation could drive an interneuron to threshold. Together, these data suggest that potent GABAergic excitation by AACs in the basal amygdala provides a mechanism for the synchronized recruitment of interneuron networks.

\section{Discussion}

We have shown that, in a population of GABAergic interneurons in the basal amygdala, single action potentials can evoke disynaptic feedforward and feedback glutamatergic EPSPs onto similar interneurons. Feedforward excitation that can drive local pyramidal neurons to threshold has recently been described for cortical axoaxonic interneurons (Szabadics et al., 2006). The fact that interneurons in the amygdala that generate disynaptic excitation express parvalbumin, and their axons make cartridge synapses suggests that they are similar to the axoaxonic interneurons in the cortex. However, in contrast to the hippocampus and cortex, in which AACs selectively innervate the axon initial segment of pyramidal neurons with cartridge synapses (Howard et al., 2005), we have shown that these interneurons in the amygdala make cartridge as well as basket synapses. Furthermore, they clearly innervate both principal neurons as well as other parvalbuminpositive interneurons. Thus, after single action potentials in a presynaptic AAC, a biphasic GABAergic-glutamatergic event sequence is evoked in postsynaptic interneurons. Our data indicate that the interneuron targets of the activated principal neurons are highly connected by both chemical and electrical synapses, suggesting that a major function of AACs may be to trigger the recruitment of homogeneous interneuron networks.

Our evidence for the recruitment of glutamatergic principal neurons as the source of the feedback EPSP is indirect. However, other possibilities for the delayed excitation can be excluded. Thus, the possibility of delayed, asynchronous corelease of both GABA and glutamate from the interneuron can be ruled out based on the sensitivity of the glutamatergic response to bicuculline, as can the possibility of direct activation of a principal neuron via the patch pipette. Rebound spiking in a principal neuron after a hyperpolarizing GABAergic IPSP (Cobb et al., 1995) is also unlikely because GABAergic IPSPs in BL principal neurons last $200 \mathrm{~ms}$ before returning to baseline. This is 2 orders of magnitude slower than the latency of feedback excitation. Because interneurons in the basal amygdala do not extend axons outside of the nucleus (Kemppainen and Pitkanen, 2000; McDonald and Betette, 2001), and the only local source of glutamate is from BL principal neurons, the glutamatergic response can only arise from local principal neurons. Furthermore, all four successfully reconstructed cells exhibiting feedback excitation had axons confined to the BL, and all exhibited closely spaced rows of boutons, or axonal cartridges. These cartridges are a hallmark of axoaxonic cells in cortex, hippocampus, and amygdala (Howard et al., 2005; Muller et al., 2005), the type of interneuron that has recently been reported to directly excite cortical layer $2 / 3$ pyramidal neurons to threshold (Szabadics et al., 2006). Thus, given the similarity of this finding to that reported for cortical AACs, and the lack of a plausible alternative explanation, we suggest that AACs of the BL can also directly depolarize local principal neurons to threshold.

Parvalbumin-expressing interneurons in the basolateral amygdala have been shown to make basket as well as cartridge synapses, and in some instances, these two synapses appear to be continuous (McDonald and Betette, 2001). However, because in both the cortex and hippocampus, AACs selectively target the axon initial segment (Howard et al., 2005), these authors concluded that these two synapses are likely to arise from different interneurons (McDonald and Betette, 2001). Our data show that single parvalbumin-expressing interneurons can form both types of synaptic contacts (Fig. 4). Although the resolution of our morphological approach does not allow us to draw a definitive conclusion, we suggest that these two structures may be continuous in the amygdala. Because interneurons that evoke GABAergic excitation form basket synapses, it is conceivable that, as well as cartridge synapses on the initial segment, basket synapses could contribute to the potent GABAergic depolarization we describe. Thus, for example, depolarizing actions of GABA have been described on adult neurons (Golding and Oertel, 1996; Gulledge and Stuart, 2003). However, perforated-patch recordings from neurons receiving somatic GABAergic input have revealed only hyperpolarizing or weakly depolarizing GABA responses from rest (Martina et al., 2001; Gulledge and Stuart, 2003; Szabadics et 
al., 2006). For somatic GABAergic inputs to drive some neurons to threshold would require the presence of a significantly reversed chloride gradient in the soma of a population of pyramidal neurons. This would need, as is seen in immature neurons, either very high expression of the $\mathrm{Na}^{+}-\mathrm{K}^{+}-2 \mathrm{Cl}^{-}$cotransporter $\mathrm{NKCC} 1$, very low expression of KCC2, or both. Although developmental studies have not shown such a distribution in adult neurons in other brain regions (Gulyas et al., 2001; Yamada et al., 2004; Takayama and Inoue, 2006), it remains possible that such a scenario is present on a subpopulation of pyramidal neurons in the basal amygdala.

Increasing evidence indicates that synaptic connectivity is not determined randomly. Reciprocally connected neurons are more common than would be expected from random connectivity (Song et al., 2005), and the strength of synapses in reciprocally connected pairs is stronger than for unidirectionally connected pairs (Yoshimura and Callaway, 2005; Yoshimura et al., 2005). Synaptically connected neurons also receive a greater degree of common input than do unconnected pairs (Song et al., 2005; Yoshimura and Callaway, 2005; Yoshimura et al., 2005). Consistent with this organization, we have shown that interneuron pairs receiving both feedforward and feedback glutamatergic excitation had a strong preference for being bidirectionally connected by GABAergic synapses. Although we could not unambiguously confirm that the same principal neuron(s) innervated the two interneurons, activation of a single AAC led to the simultaneous depolarization of downstream interneurons via at least one principal neuron intermediary. Furthermore, pairs receiving simultaneous feedforward and feedback excitation were also more likely to be electrically coupled. Together, these data suggest that one consequence of AAC activation is the recruitment of interneuron networks.

The remarkable potency of the depolarizing effect of AACs is likely to have profound implications for network activity. Although similar data are not available for the amygdala, hippocampal and cortical axoaxonic cells are known to innervate large numbers of pyramidal neurons (Howard et al., 2005), and AACs clearly provide excitation powerful enough to recruit at least some fraction of these neurons. In a study of cortical layer V pyramidal neuron connectivity, it was found that the pyramidal neuron network consisted of a skeleton of rare, strong connections that clustered together and were embed- a control

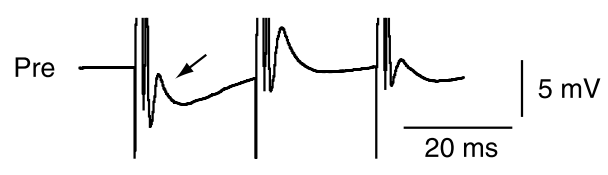<smiles>C=CC(=C)C(=C)C(=C)C</smiles><smiles>CCCCCC(C)CN[R6](=O)[O-]</smiles>

b
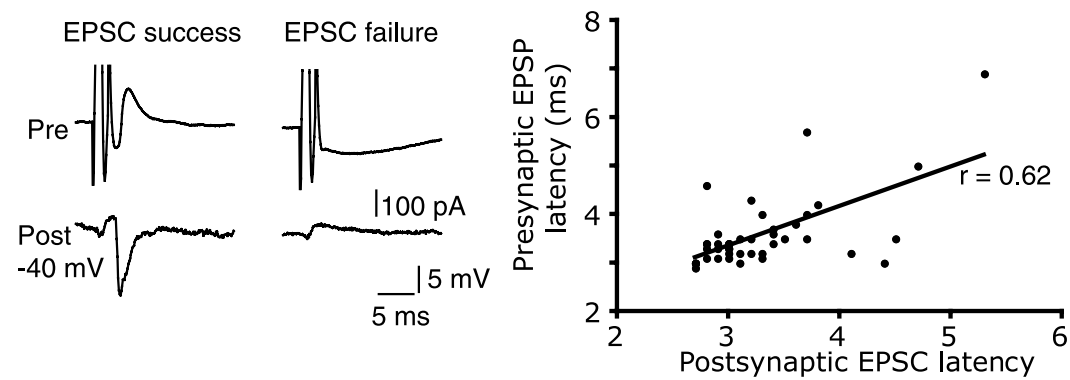

$c$
d
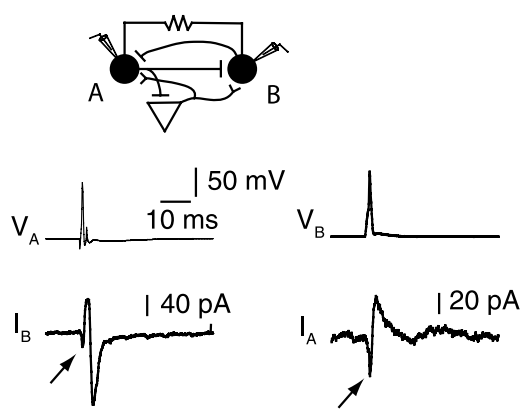

e
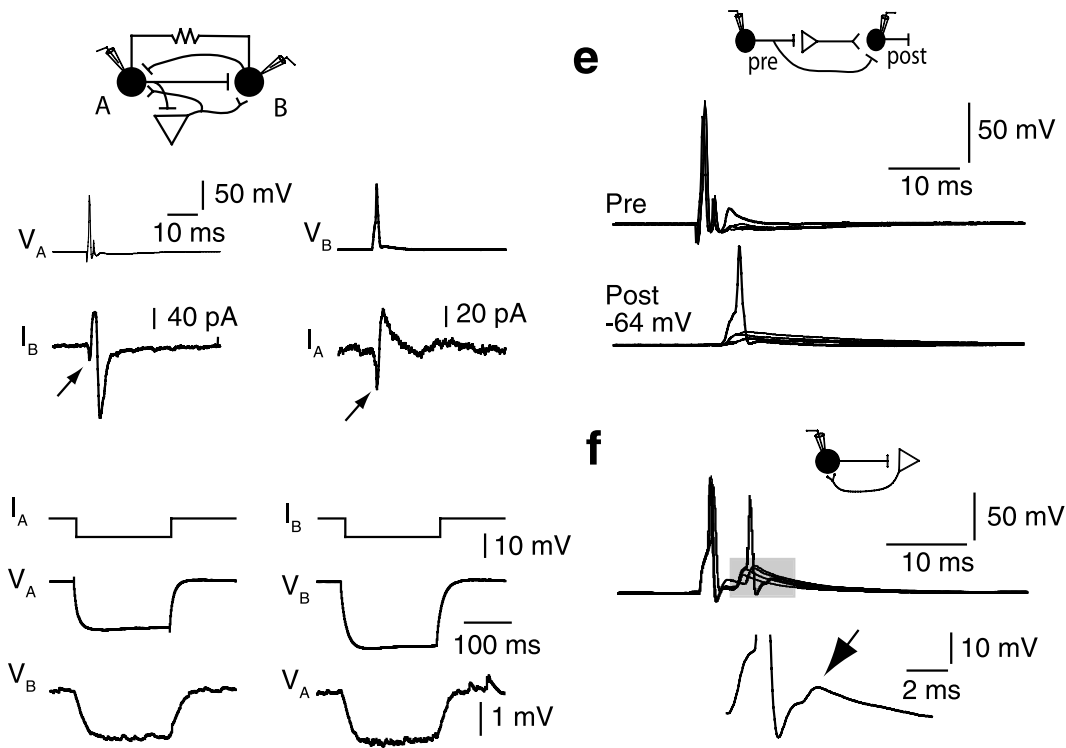

Figure 5. Excitatory interneurons drive local recurrent circuits. a, Paired recording from two EGFP-positive interneurons. A train of action potentials in the presynaptic interneuron evokes depolarizing EPSPs in both the postsynaptic and presynaptic interneurons (arrows). Application of bicuculline abolishes EPSPs in both the presynaptic and the postsynaptic neuron. Note the electrically coupled spikelets that remain in the presence of bicuculline, indicating that the two interneurons are electrically coupled. $\boldsymbol{b}$, Single glutamatergic neuron provides input to presynaptic and postsynaptic interneurons. A paired recording from two interneurons is shown. Presynaptic cell is held in current clamp, and the postsynaptic cell is voltage clamped to $-40 \mathrm{mV}$. A single action potential in the presynaptic cell evokes correlated excitation in both cells as indicated by the correlation in presynaptic and postsynaptic event latencies (graph on right). $\boldsymbol{c}, \boldsymbol{d}$, Interneuron pair in which both cells received excitation from activated principal neurons and are also connected by bidirectional GABAergic synapses ( $\boldsymbol{c}$ ) as well as by electrical synapses (d). $\boldsymbol{c}$, Traces show the response in the postsynaptic neuron to an action potential in the presynaptic neuron. An action potential in neuron $A$ evokes an outward current followed by a delayed large inward in neuron B (traces on left). An action potential in cell B evokes a GABAergic IPSC and a small glutamatergic current in cell A (traces on right). The arrows indicate voltage-clamped spikelets. $\boldsymbol{d}$, Gap junctional coupling of connected interneurons. Hyperpolarizing current injection into one cell evokes large hyperpolarization in that neuron and a smaller hyperpolarization in the electrically coupled interneuron. The presence of the gap junctional connection is seen as a "spikelet" in the postsynaptic neuron accompanying the presynaptic action potentials in c(arrows). $\boldsymbol{e}, \boldsymbol{f}$, Feedforward or feedback excitation can drive interneurons to threshold. $e$, Paired recordings from an interneuron pair in which single action potentials in the presynaptic cell evokes a delayed EPSPs in the postsynaptic interneuron driving it to threshold in some cases. $f$, Superimposed traces from a single interneuron in which a single action potentials generates feedback excitation that evokes a second interneuron spike. The shaded area is shown on an expanded scale in the bottom panel and illustrates a feedback EPSP (arrow) after the second action potential. Schematic diagrams above each figure illustrate circuit configuration. 
ded in a background of more numerous but weaker connections (Song et al., 2005). Activation of just a small number of these strong connections could lead to postsynaptic spiking. Given that these strong connections are clustered, and the highly nonrandom nature of synaptic connectivity, it is conceivable that pyramidal neurons with strong connections may be the recipient of suprathreshold AAC excitation. This provides an ideal mechanism for the rapid activation of large numbers of pyramidal neurons, as occurs in the transition from a hyperpolarized network Down state to the depolarized Up state (Steriade et al., 1993; Cossart et al., 2003; Haider et al., 2006), a phenomenon that has been observed not only in the cortex but also in the amygdala (Collins et al., 2001).

In summary, we demonstrated a powerful form of GABAergic excitation in the basal amygdala. Excitation arises from axoaxonic cells and is frequently suprathreshold to glutamatergic principal neurons, resulting in the subsequent depolarization of downstream interneurons. We show that interneurons targeted by the activated principal neuron are frequently connected by bidirectional GABAergic as well as electrical synapses, implying they belong to a single network. This form of GABAergic excitation provides an ideal means both for the repeatable activation of neuronal ensembles and for the recruitment of homogeneous interneuron networks.

\section{References}

Alger BE, Nicoll RA (1979) GABA-mediated biphasic inhibitory responses in hippocampus. Nature 281:315-317.

Alger BE, Nicoll RA (1982) Pharmacological evidence for two kinds of GABA receptor on rat hippocampal pyramidal cells studied in vitro. J Physiol (Lond) 328:125-141.

Ben-Ari Y (2002) Excitatory actions of GABA during development: the nature of the nurture. Nat Rev Neurosci 3:728-739.

Buzsaki G, Penttonen M, Nadasdy Z, Bragin A (1996) Pattern and inhibition-dependent invasion of pyramidal cell dendrites by fast spikes in the hippocampus in vivo. Proc Natl Acad Sci USA 93:9921-9925.

Chavas J, Marty A (2003) Coexistence of excitatory and inhibitory GABA synapses in the cerebellar interneuron network. J Neurosci 23:2019-2031.

Cobb SR, Buhl E, Halasy K, Paulsen O, Somogyi P (1995) Synchronization of neuronal activity in the hippocampus by individual GABAergic interneurons. Nature 378:75-78.

Collins DR, Pelletier JG, Pare D (2001) Slow and fast (gamma) neuronal oscillations in the perirhinal cortex and lateral amygdala. J Neurophysiol 85:1661-1672.

Cossart R, Aronov D, Yuste R (2003) Attractor dynamics of network UP states in the neocortex. Nature 423:283-288.

Cossart R, Bernard C, Ben-Ari Y (2005) Multiple facets of GABAergic neurons and synapses: multiple fates of GABA signalling in epilepsies. Trends Neurosci 28:108-115.

Freund TF, Buzaki G (1996) Interneurons of the hippocampus. Hippocampus 6:345-470.

Galarreta M, Hestrin S (1999) A network of fast-spiking cells in the neocortex connected by electrical synapses. Nature 402:72-75.

Gibson JR, Beierlein M, Connors BW (1999) Two networks of electrically coupled inhibitory neurons in neocortex. Nature 402:75-79.

Golding NL, Oertel D (1996) Context-dependent synaptic action of glycinergic and GABAergic inputs in the dorsal cochlear nucleus. J Neurosci $16: 2208-2219$.

Gulledge AT, Stuart GJ (2003) Excitatory actions of GABA in the cortex. Neuron 37:299-309.

Gulyas AI, Sik A, Payne JA, Kaila K, Freund TF (2001) The KCl cotransporter, KCC2, is highly expressed in the vicinity of excitatory synapses in the rat hippocampus. Eur J Neurosci 13:2205-2217.

Haider B, Duque A, Hasenstaub AR, McCormick DA (2006) Neocortical network activity in vivo is generated through a dynamic balance of excitation and inhibition. J Neurosci 26:4535-4545.

Hestrin S, Galarreta M (2005) Electrical synapses define networks of neocortical GABAergic neurons. Trends Neurosci 28:304-309.

Howard A, Tamas G, Soltesz I (2005) Lighting the chandelier: new vistas for axo-axonic cells. Trends Neurosci 28:310-316.

Kaila K, Lamsa K, Smirnov S, Taira T, Voipio J (1997) Long-lasting GABA- mediated depolarization evoked by high-frequency stimulation in pyramidal neurons of rat hippocampal slice is attributable to a networkdriven, bicarbonate-dependent $\mathrm{K}^{+}$transient. J Neurosci 17:7662-7672.

Kemppainen S, Pitkanen A (2000) Distribution of parvalbumin, calretinin, and calbindin- $\mathrm{D}(28 \mathrm{k})$ immunoreactivity in the rat amygdaloid complex and colocalization with gamma-aminobutyric acid. J Comp Neurol 426:441-467.

Magee JC, Johnston D (1997) A synaptically controlled, associative signal for Hebbian plasticity in hippocampal neurons. Science 275:209-213.

Mahanty NK, Sah P (1998) Calcium-permeable AMPA receptors mediate long-term potentiation in interneurons in the amygdala. Nature 394:683-687.

Markram H, Toledo-Rodriguez M, Wang Y, Gupta A, Silberberg G, Wu C (2004) Interneurons of the neocortical inhibitory system. Nat Rev Neurosci 5:793-807.

Martina M, Royer S, Pare D (2001) Cell-type-specific GABA responses and chloride homeostasis in the cortex and amygdala. J Neurophysiol 86:2887-2895.

Marty A, Llano I (2005) Excitatory effects of GABA in established brain networks. Trends Neurosci 28:284-289.

McBain CJ, Fisahn A (2001) Interneurons unbound. Nat Rev Neurosci 2:11-21.

McDonald AJ, Betette RL (2001) Parvalbumin-containing neurons in the rat basolateral amygdala: morphology and co-localization of Calbindin$\mathrm{D}(28 \mathrm{k})$. Neuroscience 102:413-425.

Meyer AH, Katona I, Blatow M, Rozov A, Monyer H (2002) In vivo labeling of parvalbumin-positive interneurons and analysis of electrical coupling in identified neurons. J Neurosci 22:7055-7064.

Miles R, Toth K, Gulyas AI, Hajos N, Freund TF (1996) Differences between somatic and dendritic inhibition in the hippocampus. Neuron 16:815-823.

Muller JF, Mascagni F, McDonald AJ (2005) Coupled networks of parvalbumin-immunoreactive interneurons in the rat basolateral amygdala. J Neurosci 25:7366-7376.

Muller JF, Mascagni F, McDonald AJ (2006) Pyramidal cells of the rat basolateral amygdala: synaptology and innervation by parvalbuminimmunoreactive interneurons. J Comp Neurol 494:635-650.

Pouille F, Scanziani M (2001) Enforcement of temporal fidelity in pyramidal cells by somatic feed-forward inhibition. Science 293:1159-1163.

Rivera C, Voipio J, Payne JA, Ruusuvuori E, Lahtinen H, Lamsa K, Pirvola U, Saarma M, Kaila K (1999) The $\mathrm{K}^{+} / \mathrm{Cl}^{-}$co-transporter KCC2 renders GABA hyperpolarizing during neuronal maturation. Nature 397:251-255.

Ruiz A, Fabian-Fine R, Scott R, Walker MC, Rusakov DA, Kullmann DM (2003) GABAA receptors at hippocampal mossy fibers. Neuron 39:961-973.

Smith Y, Pare JF, Pare D (1998) Cat intraamygdaloid inhibitory network: ultrastructural organization of parvalbumin-immunoreactive elements. J Comp Neurol 391:164-179.

Somogyi P, Klausberger T (2005) Defined types of cortical interneurone structure space and spike timing in the hippocampus. J Physiol (Lond) $562: 9-26$

Song S, Sjostrom PJ, Reigl M, Nelson S, Chklovskii DB (2005) Highly nonrandom features of synaptic connectivity in local cortical circuits. PLoS Biol 3:e68.

Staley KJ, Soldo BL, Proctor WR (1995) Ionic mechanisms of neuronal excitation by inhibitory GABAA receptors. Science 269:977-981.

Steriade M, McCormick DA, Sejnowski TJ (1993) Thalamocortical oscillations in the sleeping and aroused brain. Science 262:679-685.

Szabadics J, Varga C, Molnar G, Olah S, Barzo P, Tamas G (2006) Excitatory effect of GABAergic axo-axonic cells in cortical microcircuits. Science 311:233-235.

Takayama C, Inoue Y (2006) Developmental localization of potassium chloride co-transporter 2 in granule cells of the early postnatal mouse cerebellum with special reference to the synapse formation. Neuroscience, in press.

Yamada J, Okabe A, Toyoda H, Kilb W, Luhmann HJ, Fukuda A (2004) $\mathrm{Cl}^{-}$ uptake promoting depolarizing GABA actions in immature rat neocortical neurones is mediated by NKCC1. J Physiol (Lond) 557:829-841.

Yoshimura Y, Callaway EM (2005) Fine-scale specificity of cortical networks depends on inhibitory cell type and connectivity. Nat Neurosci 8:1552-1559.

Yoshimura Y, Dantzker JL, Callaway EM (2005) Excitatory cortical neurons form fine-scale functional networks. Nature 433:868-873. 